\title{
THE NOVEMBER MEETING IN SPARTANBURG
}

The four hundred ninety-sixth meeting of the American Mathematical Society was held at Wofford College, Spartanburg, South Carolina, on Friday and Saturday, November 27-28, 1953. The total attendance was over 100 , including the following 86 members of the Society:

R. W. Bagley, E. G. Begle, J. W. Brace, A. T. Brauer, E. A. Cameron, C. E. Capel, Haskell Cohen, H. S. Collins, F. E. Cothran, A. C. Downing, Jr., B. M. Drucker, L. A. Dye, S. E. Dyer, Jr., J. C. Eaves, D. O. Ellis, M. H. M. Esser, W. M. Faucett, F. A. Ficken, L. R. Ford, L. R. Ford, Jr., M. K. Fort, Jr., Tomlinson Fort, H. K. Fulmer, Seymour Ginsburg, Wallace Givens, R. A. Good, W. L. Gordon, E. E. Grace, J. S. Griffin, Jr., H. C. Griffith, E. H. Hadlock, B. F. Hadnot, S. W. Hahn, Dick Wick Hall, E. A. Hedberg, Nickolas Heerema, R. T. Herbst, P. S. Herwitz, J. G. Horne, Jr., A. S. Householder, G. B. Huff, S. L. Jamison, R. J. Koch, F. W. Kokomoor, G. B. Lang, J. W. Lasley, Jr., T. H. Lee, F. A. Lewis, R. A. Lytle, C. W. McArthur, J. S. MacNerney, Nathaniel Macon, W. R. Mann, D. D. Miller, J. C. Morelock, J. A. Nohel, F. R. Olson, W. V. Parker, S. E. Pence, B. J. Pettis, C. M. Petty, E. E. Posey, J. H. Roberts, R. A. Roberts, W. C. Royster, B. M. Seelbinder, A. L. Shields, J. R. Shoenfield, W. S. Snyder, G. L. Spencer, H. D. Sprinkle, W. L. Strother, P. M. Swingle, H. E. Taylor, J. M. Thomas, H. E. Vaughan, T. L. Wade, Jr., A. D. Wallace, M. J. Walsh, H. C. Wang, A. E. Welch, G. T. Whyburn, L. S. Whyburn, W. M. Whyburn, R. A. Willoughby, C. T. Yang.

By invitation of the Committee to Select Hour Speakers for Southeastern Sectional Meetings, Professor B. J. Pettis of Tulane University and Dr. A. S. Householder of the Oak Ridge National Laboratory addressed the Society. Dr. Householder's address, entitled Generation of errors in digital computation, was delivered at 8:00 P.M. on Friday, with Professor Wallace Givens presiding. The address by Professor Pettis, entitled Certain aspects of linear topological spaces, was given at 10:00 A.M. on Saturday, with Professor G. T. Whyburn presiding.

Four sessions for the presentation of contributed papers were held on Friday afternoon and Saturday morning. Presiding officers for these sessions were Professors Tomlinson Fort, W. V. Parker, F. A. Lewis, and F. W. Kokomoor.

All sessions were held in the Whitefoord Smith Library on the campus of Wofford College. Members of the Wofford College faculty and their wives were hosts at coffee hours on Friday morning, Friday afternoon, and Saturday morning. President Pendleton Gaines of Wofford College extended a cordial welcome to the Society at its opening session on Friday. He and Mrs. Gaines entertained with a reception in the president's home on Saturday morning. A resolution 
expressing the appreciation of the Society to its hosts was offered by Professor G. B. Huff and unanimously adopted.

Abstracts of the papers presented follow below. Abstracts whose numbers are followed by the letter " $t$ " were presented by title. In the case of joint papers, (p) following one of the authors' names indicates the one who actually read the paper. Mr. McAuley was introduced by Professor Jones, Mr. C. W. Huff by Professor G. B. Huff, Professor Ohtsuka by Professor Lohwater, Dr. Pohrer by Professor Roberts, Miss Norton by Professor Miles, Mr. Aucoin by Professor Wang, Dr. Saaty and Mr. Gass by Professor Whyburn.

\section{Algebra ANd Theory of Numbers}

54. A. T. Brauer and B. M. Seelbinder (p): On a problem of partitions. II.

Let $a_{1}, a_{2}, \cdots, a_{k}$ be relatively prime positive integers. Let $d_{r}$ be the greatest common divisor of $a_{1}, a_{2}, \cdots, a_{r}$ for $r=1,2, \cdots, k$. It was proved in part I (Amer. J. Math. vol. 64, pp. 299-312) that the Diophantine equation $a_{1} x_{1}+a_{2} x_{2}+\cdots+a_{k} x_{k}$ $=n$ always has solutions in positive integers $x_{1}, x_{2}, \cdots, x_{k}$ if $n>T=T\left(a_{1}, a_{2}, \cdots, a_{k}\right)$ $=a_{2}\left(d_{1} / d_{2}\right)+a_{3}\left(d_{2} / d_{3}\right)+\cdots+a_{k}\left(d_{k-1} / d_{k}\right)$. Moreover it was proved that $T$ is the smallest possible bound for the solution of the given equation by positive integers if for $r=3,4, \cdots, k$ the numbers $a_{r} d_{r-1} / d_{r}$ can be written in the form $a_{r} d_{r-1} / d_{r}=a_{1} y_{r 1}$ $+a_{2} y_{r 2}+\cdots+a_{r-1} y_{r, r-1}$, where all the $y^{\prime} s$ are non-negative integers. For $k=3$ the condition was proved necessary. It is proved in this paper that this condition is necessary for every $k$. If $T$ is not the best bound, then $T-\min \left(a_{1}, a_{2}, \cdots, a_{k}\right)$ is a bound for the solution of the given equation by positive integers. (Received September 21, 1953.)

55t. Leonard Carlitz: An application of the multiplication formulas for the Jacobi elliptic function.

Put sn $t x / t$ sn $x=\sum_{0}^{\infty} \beta_{2 m}(t) x^{2 m} /(2 m)$ !, where $t$ is an odd integer and $u=k^{2}$ is an indeterminate; also put $\mathrm{sn} x=\sum_{0}^{\infty} A_{2 m+1}(u) x^{2 m+1} /(2 m+1)$ !. It is proved that $\beta_{2 m}(t)$ $=H_{m}(t)-\sum A^{m /(p-1)}(u) / p$, where the summation is over all primes $p$ such that $p \mid t$ and $p-1 \mid 2 m$, and $H_{m}(t)$ is a polynomial in $u$ with integral coefficients. It is also proved that $t \sum_{s=0}^{r}(-1)^{r-\bullet} C_{r, 8} \beta_{m+8(p-1)}(t) A_{p}^{r-1}(u) \equiv 0\left(\bmod p^{m}, p^{r}\right)$ for $p>2, r \geqq 1$. (Received October $5,1953$.

56t. Leonard Carlitz: Congruences for the solutions of certain difference equations of the second order.

D. H. Lehmer (Ann. of Math. vol. 33 (1932) pp. 143-150) discussed the difference equation $u_{n+1}=(a n+b) u_{n}+c u_{n-1}$. In particular he proved that if $(a, m)=1$, then $a_{n+2 m} \equiv c^{m} a_{n}(\bmod m) .1$. In the present paper, taking the equation in "normal" form $u_{n+1}=n u_{n}+c u_{n-1}$, with $c$ and $c^{-1}$ prime to $m$, it is shown that $\Delta^{2 r-1} u_{n} \equiv \Delta^{2 r} u_{n} \equiv 0$ $\left(\bmod m^{r}\right)$ for $r \geqq 1$ and all $n$, where $\Delta^{r} u_{n}=\sum_{c-0}^{r}(-1)^{r-s} C_{r, 8} c^{m(r-s)} u_{n+2 m s}$; for $m$ even the modulus is $2^{-r+1} m^{r}$. 2. Turning next to the nonhomogeneous equation $u_{n+1}$ $=n u_{n}+b c u_{n-1}+b^{n}+c^{n}$ with $u_{0}=0, u_{1}=1$, we assume that $b \equiv c(\bmod p)$ for each prime $p$ dividing $m$ and show that $\Delta^{2 r} u_{n} \equiv \Delta^{2 r+1} \equiv 0\left(\bmod m^{r} m^{\prime}\right)$, where $m=\prod p^{p}, m^{\prime}=\prod p^{r-1}$, 
$m$ odd, and now $\Delta^{r} u_{n}=\sum(-1)^{r-} C_{r, c^{m}} c^{m(r-s)} u_{r+m s .}$ The nonhomogeneous case includes the so-called menage polynomials. We remark that in the proof of these results it seems desirable to let $n$ take on negative values. (Received October $5,1953$. )

57t. Leonard Carlitz: Congruence properties of the menage polynomials.

If $U_{n}=U_{n}(t)$ denotes the menage polynomials and $\Delta f_{n}=f(n+m)-(t-1)^{m} f(n)$, $\Delta^{r} f(n)=\Delta \cdot \Delta^{r-1} f(n)$, it is proved that (1) $\Delta U_{n} \equiv 0\left(\bmod m^{\prime}\right)$ and $(2) \Delta^{2 r} U_{n} \equiv \Delta^{2 r+1} U_{n} \equiv 0$ $\left(\bmod m^{r} m^{\prime}\right)$, where $m^{\prime}$ is defined by $m=\prod p^{e}, m^{\prime}=\prod p^{\sigma-1}, m$ odd. For previous results see J. Riordan, The arithmetic of menage numbers, Duke Math. J. vol. 19 (1952) pp. 27-30 and L. Carlitz, Congruences for the menage polynomials, Duke Math. J. vol. 19 (1952) pp. 549-552. (Received October 5, 1953.)

58t. Leonard Carlitz: Congruence properties of the polynomials of Hermite, Laguerre and Legendre.

For the Hermite polynomial $H_{n}(x)$ it is proved that (1) $H_{n+m}(x) \equiv(2 x)^{m} H_{n}(x)$ $(\bmod m)$, where $m$ is an arbitrary integer, and $(2) \Delta^{2 r} H_{n}(x) \equiv \Delta^{2 r-1} H_{n}(x) \equiv 0\left(\bmod m^{r}\right)$ for all $r \geqq 1$, where $\Delta^{r} H_{n}(x)=\sum_{s-0}^{r}(-1)^{r-s} C_{r, s} H_{n+s m}(x) H_{(r-s)}(x)$. Similar results are proved for the polynomial $\Lambda_{n}^{(\alpha)}(x)=n ! L_{n}^{(\alpha)}(x)$, where $L_{n}^{(\alpha)}(x)$ denotes the general Laguerre polynomial. For the Legendre polynomial $P_{n}(x)$ it is proved that $n ! P_{n+m}(x)$ $\equiv P_{n}(x) P_{m}(x), n ! P_{m-n-1}(x) \equiv n ! P_{n}(x) P_{m-1}(x)(\bmod m)$; like results hold for the ultraspherical polynomials. (Received October $5,1953$.

59t. Leonard Carlitz and F. R. Olson: Some theorems on Bernoulli and Euler numbers of higher order.

Employing the notation of Nörlund's Differenzrechnung, Chap. 6, for the numbers $B_{m}^{(k)}\left[\omega_{1}, \cdots, \omega_{k}\right]$, etc., congruences are proved that generalize theorems of Glaisher and Nielsen. Similar theorems are proved for the numbers of negative order as well as for numbers of "mixed" order that include both those of positive and negative order. Finally most of the results are extended to the case of more general sequences. (Received August 24, 1953.)

60. R. A. Good, III and D. W. Hall (p): On a class of orthogonal matrices.

We study the class $\left\{Q_{n}\right\}$ of all orthogonal matrices of order $n$ with rational elements, with determinant one, and with the additional property that the sum of the elements in each row and each column is one. The determination of all members of the class $\left\{Q_{n}\right\}$ may be reduced to the search for all positive rational automorphs of a certain quadratic form. Using this approach and other special techniques, we give a parametrization of the members of each class $\left\{Q_{n}\right\}$ for $n \leqq 5$. (Received October 14, 1953.)

61. E. H. Hadlock: Ternary quadratic forms equivalent to forms with one term of type $2 b_{i j} y_{i} y_{j}, j \neq i$.

Let $\left(c_{i j}\right)$ be a matrix of determinant one which takes the ternary quadratic form $f=\sum x_{i} X_{i}$, with determinant not equal to zero, into an equivalent form $f^{\prime}$ containing one term of type $2 b_{i j} b_{i} b_{j}, j \neq i$. The elements of the third column of $\left(c_{i j}\right)$ are arbitrary, except that their g.c.d. is one. All integral solutions of a linear homogeneous equation, 
involving as variables the elements of the first column of $\left(c_{i j}\right)$, yield all values for which $b_{13}=0$. Similarly, values of the elements of the second column are restricted so that $b_{23}=0$. It is found that a necessary and sufficient condition that $f$ be equivalent to $f^{\prime}$ is that $f$ represents, primitively, for certain values of the variables, a divisor of $g$, the g.c.d. of the three linear functions $X_{i}$ for those same values of the variables. Moreover it is necessary that this $g$ be a divisor of the determinant of $f$. (Received October 14, 1953.)

62. C. W. Huff: On pair of matrices (of order two) $A, B$ satisfying the condition $e^{A} e^{B}=e^{A+B} \not e^{B} e^{A}$.

M. Fréchet has pointed out [Rend. Circ. Mat. Palermo (1) vol. 2 (1953); see also Mathematical Reviews vol. 14 (1953) p. 237] that in his attack on pairs of matrices $X, Y$ satisfying the equation $e^{X} e^{Y}=e^{X+Y}$ [Rend. Circ. Mat. Palermo (1) vol. 1 (1952) ], he had actually described pairs satisfying the relationship $e^{X} e^{Y}=e^{X+Y}=e^{Y} e^{X}$. This paper completes Fréchet's work in the following way. Let $(A, B)$ have property $Q$ mean that $e^{A} e^{B}=e^{A+B} \neq e^{B} e^{A}$. Let $A^{\prime}$ be the transpose of the matrix $A$. Then an equivalence relation, $\sim$, is defined by $(A, B) \sim(X, Y)$ means that there exists a nonsingular matrix $C$ and scalar matrices $H$ and $K$ such that either $X=C(A+H) C^{-1}$ and $Y=C(B+K) C^{-1}$ or $X=C\left(B^{\prime}+H\right) C^{-1}$ and $Y=C\left(A^{\prime}+K\right) C^{-1}$. Two theorems are established: (1) If $(X, Y)$ has property $Q$ then there exists a pair of matrices $A$ and $B$ such that $A=\left(\begin{array}{l}x \\ 0 \\ 0\end{array}\right), x \neq 0$, and $B=\left(\begin{array}{l}0 \\ 0\end{array} ;\right), g \neq 0$, and $(X, Y) \sim(A, B)$. (2) If $(z)=\left(e^{z}-1\right) / z$ for $z \neq 0$ and $f(0)=1, A=\left(\begin{array}{l}z \\ 0 \\ 0\end{array}\right), x \neq 0$, and $B=\left(\begin{array}{l}0 \\ 0 \\ 0 \\ 0\end{array}\right)$, then $(A, B)$ has property $Q$ if, and only if, $b \neq 0, f(y)=f(y-x)$ and $f(x) f(y) \neq 0$. (Received October 15, 1953.)

63t. H. L. Lee: On a certain congruence of variable degree. Preliminary report.

The congruence $x^{x-1} \equiv 1(\bmod p)$ where $p$ is a prime is considered. A sufficient condition is obtained for the solution of this congruence, viz. $p=n a^{n}-n+1$ with $x=a^{n}$. By the use of the primitive root $g$ of $p$, it is found that a necessary and sufficient condition that the congruence have a solution is that for $g^{k} \equiv s(\bmod p), p-1 \mid k(s-1)$. (Received October 16, 1953.)

\section{T. H. Lee: Similarity of matrices with rational generalized qua- ternions as elements.}

Let $\alpha$ and $\beta$ be square-free rational integers and $r_{0}, r_{1}, r_{2}$, and $r_{3}$ rational numbers. We define $i$ and $j$ by the equations $i^{2}=-\alpha, j^{2}=-\beta$, and $i j=-j i$. The elements $r=r_{0}+r_{1} i+r_{2} j+r_{3} i j$ form a rational generalized quaternion algebra $Q(\alpha, \beta)=Q$. C. G. Latimer (Quaternion algebras, Duke Math. J. vol. 15 (1948) pp. 357-366) has shown that there are infinitely many distinct rational generalized quaternion algebras $Q$, and all except one (which we shall denote by $Q_{-1}$ ) are division algebras. In this paper, matrices $A$ whose elements belong to a rational generalized quaternion algebra $Q$, with $Q \neq Q_{-1}$, are studied. Invariant factors are defined and shown to be rational polynomials. A necessary and sufficient condition for similarity (with respect to rational generalized quaternions) of matrices with elements in $Q$ is that the two matrices have the same invariant factors. This is a generalization of the work of Louise A. Wolf (Similarity of matrices in which the elements are real quaternions, Bull. Amer. Math. Soc. vol. 42 (1936) pp. 737-743). But the proof given here is different from that of Miss Wolf's proof in that a method is given for constructing the transforming matrix for the similarity. (Received October 8, 1953.) 
65. F. R. Olson: Arithmetic properties of Bernoulli numbers of higher order.

L. Carlitz (Quart. J. Math. Oxford Ser. (2) vol. 4 (1953) pp. 112-116) has proved $B_{p+2}^{(p+1)} \equiv p^{3} / 6\left(\bmod p^{4}\right)$ and $B_{p}^{(p)} \equiv\left(-p^{2} / 2\right)(p-1) !\left(\bmod p^{5}\right)$ for primes $p \geqq 5$. These results are sharpened to $B_{p+2}^{(p+1)} \equiv p^{4} / 4-\left(p^{3} / 6\right)(p-1) !\left(\bmod p^{5}\right), p \geqq 5$, and $B_{p}^{(p)}$ $\equiv\left(-p^{2} / 2\right)(p-1) !+\left(p^{5} / 36\right) B_{p \rightarrow 3}\left(\bmod p^{6}\right), p \geqq 7$. Like congruences are obtained for generalized Bernoulli numbers $B_{n}^{(k)}\left[\omega_{1}, \omega_{2}, \cdots, \omega_{k}\right]$, Nörlund, Differenzrechnung, Chap. 6, which apply to numbers of either positive or negative order and indeed to the numbers of "mixed" order discussed in Some theorems on Bernoulli and Euler numbers of higher order, Bull. Amer. Math. Soc. Abstract 60-1-59. (Received August 24, 1953.)

\section{W. V. Parker: $A$ note on normal matrices.}

In an earlier paper [Duke Math. J. vol. 15 (1948) pp. 439-442] the author proved: If $A=\left(a_{i j}\right)$ is a normal matrix and the characteristic function of $A$ is $\Pi\left(x-a_{i i}\right)$, then $A$ is a diagonal matrix. This result is extended to give the following: If a normal matrix $A$ can be partitioned into a block matrix in such a way that the characteristic function of $A$ is the product of the characteristic functions of the diagonal block, than $A$ is a diagonal block matrix. (Received October 15, 1953.)

\section{R. G. Pohrer: On some types of equations in a finite field.}

The author classifies several types of equations in a finite field according to the nature of the number of solutions. By direct methods, the number of solutions of equations formed from various combinations of these types, among themselves and conjoined with more general kinds, can be determined. Special examples include equations formed from combinations of quadratic forms, as $c_{1} Q_{1}\left(x_{1}\right) Q_{2}\left(x_{2}\right)+\cdots$ $+c_{8} Q_{2 s-1}\left(x_{2 s-1}\right) Q_{28}\left(x_{2 s}\right)=\alpha$, where $\alpha, c_{i} \in G F(q), q=p^{n}, Q_{2 i-1}\left(x_{2 i-1}\right), Q_{2 i}\left(x_{2 i}\right)$ are $2 s$ quadratic forms $(i=1,2, \cdots, s)$; the results hold for $q$ odd and even. Simple combinations of Moore determinants, considered as equations over the $G F(q)$, can also be treated, as $c_{1} D_{1}\left(x_{1}\right)+\cdots+c_{s} D_{s}\left(x_{s}\right)=\alpha$, where $\alpha \in G F(q), c_{i} \in G F(p), D_{i}\left(x_{i}\right)$ are $s$ Moore determinants $(i=1,2, \cdots, s)$. (Received October 13, 1953.)

\section{R. A. Willoughby: A note on functional completeness.}

Let $F=\{0,1,2, \cdots, n-1\}$ where $n \geqq 2$ and let $\times$ be a multiplication such that (0) if $a, b \in F$ then $a \times b \in F$, (1) $a \times b=b \times a$, (2) $(a \times b) \times c=a \times(b \times c),(3) a \times 1=1 \times a$ $=a$, (4) $a \times 0=0 \times a=0$. Suppose that $F$ can be partitioned into two sets $F_{1}$ and $F_{2}$ such that (5) $1 \in F_{1},(6) 0 \in F_{2},(7)$ if $a, b \in F_{1}$ then $a \times b \in F_{1}$, (8) there exists a $c \in F_{1}$ and a positive integer $m$ such that $(c \times b)^{m}=0$ for all $b \in F_{2}$, (9) either $\left(c \times \prod_{a \in F_{1}} a\right)^{m}=1$ or $F_{2}$ contains a nonzero nilpotent element or $F_{1}$ contains at least three distinct elements. Then, there exists a $0 \rightarrow 1$ transitive permutation on $F, \Delta$, such that $(F, x, \Delta)$ is functionally complete. Multiplication modulo $n$ satisfies (0)-(9) above. (Received September 14, 1953.)

\section{ANALYSIS}

69t. J. H. Barrett: Differential equations of non-integer order. II. Properties of solutions.

Let $\alpha$ be a positive real number; $n$ be the positive integer such that $n-1<a<n$; $I(-\alpha ; a, x \mid f)=D_{x} I(n-\alpha ; a, x \mid f)$, where $I(\beta ; a, x \mid f)=\int_{a}^{z} f(t)\left((x-t)^{\beta-1} / \Gamma(\beta)\right) d t$ for 
$\beta>0$. Then there is a unique solution $Y_{\alpha}(x)$ of the differential equation $I(-\alpha ; 0, x y)$ $+y(x)=0, x>0$, which satisfies the boundary condition $I\left(1-\alpha ; 0,0^{+} \mid y\right)=1$ and, if $n>1$, the additional conditions $I\left(i-\alpha ; 0,0^{+} \mid y\right)=0, i=2,3, \cdots, n$. For $0<\alpha<1$ and $x>0, Y_{\alpha}(x)$ satisfies the following relationships, also satisfied by $Y_{1}(x)=e^{-x}: Y(x)>0$; $\int_{0}^{\infty} Y(t) d t=1$ and $\lim _{x \rightarrow \infty} I\left(\beta ; 0, x \mid Y_{\alpha}\right)=0$ for $0<\beta<1$. These results are applied to the complex entire function $E_{\alpha}(z)=\sum_{p-0}^{\infty} z^{p} / \Gamma(p \alpha+1)$ to show that it does not vanish, is less than 1 , and $D_{x} E_{\alpha}(x)>0$ on the negative real axis. For $\alpha>1$, results of Mittag-Leffler and Wiman (Acta Math. vol. 29) are used to show that for $1<\alpha<2$ and $x>0, Y_{\alpha}(x)$ has only a finite number of zeros and for $\alpha>2$ that $Y(x)$ is oscillatory. (Received October 13, 1953.)

70. Seymour Ginsburg: On the $\lambda$-dimension and the A-dimension of partially ordered sets.

In this paper the $\lambda$-dimension (Komm, On the dimension of partially ordered sets, Amer. J. Math. vol. 70 (1948) pp. 507-520) of the partially ordered set consisting of sequences of real numbers, ordered as follows, is shown to be $\aleph_{0} \cdot\left(x_{1}, \cdots, x_{n}, \cdots\right)$ $\leqq\left(y_{1}, \cdots, y_{n}, \cdots\right)$ if and only if $x_{n} \leqq y_{n}$ for all $n$. A new kind of dimension is also discussed. The linear order $L$ on an abstract set $S$ is called an $A$-extension of the partial order $P$ on $S$ if $L$ is an extension of $P$ which is similar to a set having property $A$. By the $A$-dimension of a partial order $P$ on $S$ is meant the minimum number of $A$-extensions of $P$ which realize $P$. A nontrivial example is given to show that the $\lambda$-dimension and the $A$-dimension need not be equal, even when both dimensions exist. (Received October 13, 1953.)

\section{B. F. Hadnot: Note on convergence of a class of series.}

R. D. Carmichael has established [Trans. Amer. Math. Soc. vol. 17, pp. 218-220] convergence theorems for $D(z)=\sum_{n=0}^{\infty} C_{n} \exp \left(\lambda_{1 n} z+\lambda_{2 n} z^{2}+\cdots+\lambda_{t n} z^{t}\right)$ where the $\lambda$ 's are subject to severe conditions. The author has established convergence theorems for $\vartheta(z)=\sum_{n-0}^{\infty} C_{n} \exp \left(\lambda_{1 n} W_{1}(z)+\lambda_{2 n} W_{2}(z)+\cdots+\lambda_{t n} W_{t}(z)\right)$ for more general $\lambda^{\prime} \mathrm{s}$ and arbitrary functions $W_{j}(z)$. (Received October 13, 1953.)

\section{P. S. Herwitz: On the approximate solutions of differential equa-} tions by difference equations.

This paper considers round-off and truncation errors which arise when differential systems are approximated by difference systems in the manner described by W. M. Whyburn in Amer. J. Math. vol. 51, pp. 265-269. The Picard method of successive approximations with variations due to Moulton and others is used, and questions of stability for the numerical solutions are investigated. (Received October 14, 1953.) tions.

73. J. S. MacNerney: Hellinger integrals and Stieltjes integral equa-

Each of $m$ and $n$ is a real, continuous, nondecreasing, not constant function on the number interval $[0,1]$. The Hellinger integral $\int_{x}^{y}[d f d g] / d m$ is developed as a tool for studying the system $\left(^{*}\right) A(x)-A(0)=\int_{0}^{*} B d m, B(x)-B(0)=\int_{0}^{x} A d n-z \int_{0}^{x} A d h$ $+C(0)-C(x)$ with various two-point boundary conditions on $A$ and $B$ ( $h$ continuous and increasing, $C$ continuous and of bounded variation). The study of the integral provides an elementary proof that there are uncountably many mutually exclusive (except for the origin) linear manifolds in Hilbert space, each dense in the space. 
Solutions of (*) are provided by a general existence theorem due to Wall (Rend. Circ. Mat. Palermo (2) vol. 2, (1953)). Boundary value problems are studied by introducing a resolvent pair $K, L$ for $\left({ }^{*}\right)$ with each set of boundary conditions $-K$ behaves as a Green's function. Expansion theorems are obtained as follows: the Hellinger integral is used to define an inner product function $Q$; for each set of boundary conditions there is found a Hilbert space $M$ (of continuous functions), with inner product $Q$, in which the corresponding $K$ is the reproducing kernel; orthogonal eigenfunctions of $K$, suitably normalized, also form an orthonormal set relative to $Q$ and this set is complete in $M$. (Received October 13, 1953.)

74t. J. S. MacNerney: Note on a functional Hilbert space. Preliminary report.

Each of $m$ and $n$ is a continuous function from the real numbers to the real numbers which is nondecreasing and not constant; $Q[f, g]=\int_{-\infty}^{+\infty}\left[d f d g^{*}\right] / d m+\int_{-\infty}^{+\infty}\left[f g^{*}\right] d n$ for all ordered pairs $f, g$ of functions from the real to the complex numbers for which these integrals exist (see preceding abstract); $M$ is the linear orthogonal space of all $f$ such that $Q[f, f]$ exists, with inner product function $Q$. It is proved that $M$ is a Hilbert space of continuous functions with a continuous reproducing kernel $K$, and there is paired with $K$ a function $L$ such that, if $C$ is continuous and of bounded variation on each number interval then, in order that, for each $s, A(s)=\int_{-\infty}^{+\infty} K(s, t) d C(t)$ and $B(s)=\int_{-\infty}^{+\infty} L(s, t) d C(t)$, it is necessary and sufficient that (1) $\int_{x}^{\infty} B d m=A(y)-A(x)$ and $\int_{x}^{y} A d \bar{d} n=B(y)-B(x)+C(y)-C(x)$ for all $x$ and $y$ and (2) $A(x) L(x, s)-B(x) K(x, s)$ $\rightarrow 0$ as $x \rightarrow-\infty$ and as $x \rightarrow+\infty$ for each number $s$. (Received October 13,1953.)

\section{J. A. Nohel: Stability of perturbed periodic motions.}

In the nonlinear system (1) $x^{\prime}=A x+\mu f(x, t, \mu)\left({ }^{\prime}=d / d t\right), x$ is a real vector of $n$ components, $A$ is a real constant $n \times n$ matrix, $\mu$ is a real parameter, and $f$ is a real vector of $n$ components with period $2 \pi$ in $t(2 \pi$ need not be the least period). Let the unperturbed linear system have a real periodic solution $x=p(t)$ with period $2 \pi$. Sufficient conditions for the asymptotic stability of the periodic solution of (1) $x=q(t, \mu)$ which (i) has period $2 \pi$ in $t$, (ii) is continuous for all $t$ and $|\mu|$ sufficiently small, and (iii) for which $q(t, 0)=p(t)$, are established for $\mu>0$ and sufficiently small. Such a solution exists by Theorem 1 of E. A. Coddington and N. Levinson (Perturbations of linear systems with constant coefficients, Annals of Mathematics Studies, no. 29, pp. 19-35, Princeton, 1952). In case system (1) is autonomous, the orbital stability of the continuous periodic solution $x=q(t, \mu)$ (Ibid., Theorem 4) with period $2 \pi+\tau(\mu)$, where $\tau(0)=0$ and $q(t, 0)=p(t)$, is investigated for $\mu>0$ and sufficiently small. (Received October 7, 1953.)

76t. Makoto Ohtsuka: Note on functions bounded and analytic in the unit circle.

W. Gross [Math. Ann. vol. 79 (1918) pp. 201-208] gave an example of an entire function $w=\phi(z)$ with the property that, for any complex number $w$, there exists an uncountable set of asymptotic paths along which $\phi(z)$ tends to $w$. By conformal mapping it is then a simple matter to give an example of a function analytic in the unit circle $|z|<1$ with a similar property in the neighborhood of a point $z_{0}$ of $|z|=1$. If the function is required to be bounded, however, it can have at most one asymptotic value at a point of $|z|=1$. It is proved that there exists a function $w=f(z)$, analytic and bounded in $|z|<1$, such that $f\left(e^{i \theta}\right)=\lim _{r \rightarrow 1} f\left(r e^{i \theta}\right)$ has modulus 1 for 
almost all $e^{i \theta}$ on $|z|=1$, and such that, for any $\alpha$ with $|\alpha| \leqq 1$, the equation $f\left(e^{i \theta}\right)=\alpha$ is satisfied on an uncountable set of $e^{i \theta}$ on $|z|=1$, i.e. that the radial limit values of $f(z)$ at a set of linear measure zero on $|z|=1$ fills the open set $|z|<1$. (Received October 7, 1953.)

\section{W. C. Royster: Note on values omitted by p-valent functions.}

Let $\mathcal{F}$ be the class of $p$-valent functions $w=f(z), p$ a positive integer, which are regular in $|z|<1$ and have a $p$-tuple zero at the origin. Hayman (Symmetrization in the theory of functions) states that if $f(z) \in \mathcal{F}$ then $f(z)$ takes on every value in $|w|<(1 / 4)^{p}$ exactly $p$ times. This result is sharp. Using this result and a boundedness condition, one proves that if $f(z) \in \mathcal{F}$ omits a value $\alpha$ at least once for $z$ in $|z|<1$, then a lower bound for $|\alpha|$ is found in sharp form. Also using a theorem due to Biernacki (Bull. Sci. Math. (2) vol. 70) a lower bound for $|\alpha|$ is found in sharp form if $f(z)$ omits a value $\alpha$ exactly $p$ times. By constructing bounded functions from $\mathcal{F}$ and applying the above results other theorems are proven. (Received October 14, 1953.)

\section{A. L. Shields: Measure on a projective limit.}

Let $A$ be a directed set, and for each $a \in A$ let $\left(X_{a}, S_{a}, m_{a}\right)$ be a $\sigma$-finite measure space. For each pair $a, b \in A$ such that $a \leqq b$ there is a function $\phi_{a b}$ from $X_{b}$ to $X_{a}$ such that, if $E \in S_{a}$, then $\phi_{a b}^{-1}(E) \in S_{b}$, and $m_{a}(E)=m_{b}\left(\phi_{a b}^{-1} E\right)$. Let $X$ be the projective limit of the spaces $X_{a}$ under the maps $\phi_{a b}$. Let $E_{a} \in S_{a}$ for some fixed $a$. Let $\left[E_{a}\right]$ be the set of all those points of $X$ whose $a$ th coordinate is in $E_{a}$. The sets $\left[E_{a}\right]$ form a ring. If we define $m\left(\left[E_{a}\right]\right)=m_{a}\left(E_{a}\right)$ this is a $\sigma$-finite measure on the ring, hence can be extended to the $\sigma$-ring generated by the ring. In case the $X_{a}$ and $X$ are locally compact groups, $m_{a}$ is Haar measure, $\phi_{a b}$ are homomorphisms, then the domain of definition of the completion of $m$ is the Borel field in $X$, and $m$ is a Haar measure on $X$. (Received November 27, 1953.)

\section{J. M. Thomas: Reflex solutions of linear differential equations.}

The term "reflex functions" will describe the class of even and odd functions and "equation" will mean "ordinary linear homogeneous differential equation." An equation is reflex if its coefficients are alternately even and odd, the initial being unity. A reflex equation of order $n$ has both an even solution with $[(n+1) / 2]$ arbitrary constants and an odd solution with $[n / 2]$. If each coefficient in an equation is replaced by its even or by its odd part, the choice alternating from term to term, two equations $M_{0}, M_{1}$ of orders $n$ and at most $n-1$ respectively result. $M_{0}$ is reflex and $M_{1}$ can be made reflex in a region where its initial does not vanish. The system $M_{0}, M_{1}$ is satisfied by the reflex solutions of the original equation, the even corresponding to initial determinations with derivatives of odd order zero. The system $M_{0}, M_{1}$ can be applied to determine all equations with reflex solutions. Extension is made to the case where the zero of the independent variable is singular for the equation. This involves modifying the form of the initial determination and the number of arbitrary constants. (Received October 14, 1953.)

\section{Applied Mathematics}

80. F. A. Ficken: Reduction of a nonlinear parabolic problem with piecewise constant coefficients. Preliminary report. 
A problem consisting of $(E)\left[u_{x}-\psi u(1-u)-p u\right]_{x}=h u_{t}(p, \psi, h$ constant), an initial condition, and certain end conditions at $x=0$ and $x=N$ has been linearized by Montroll and Newell (Journal of Applied Physics vol. 23 (1952) pp. 184-194). When $\psi, p$, and $h$ are step-functions with jumps at $x=j_{k}\left(\left(j_{0}=\right) 0<j_{1}<\cdots<j_{n-1}\right.$ $<N\left(=j_{n}\right)$ ), standard junction conditions ( $u$ continuous, $u_{x}$ jumps) do not linearize directly. Now $u_{k}$ is expressed for $j_{k-1}<x<j_{k}$ in terms of a new unknown $z_{k}$ satisfying a linear homogeneous $\left(E_{k}^{*}\right)$ and a function $\theta_{k}(t)$ satisfying $\left(\lambda_{k-1}\right) h_{k} \dot{\theta}_{k}=l_{k-1} z_{k}$, where $l_{k-1} z_{k}$ is a linear combination of $z_{k}$ and $z_{k x}$ at $j_{k-1}$. By using the jump condition at $j_{k}$ $(1 \leqq k \leqq n-1)$ one obtains a function $\phi_{k}=c_{k} \theta_{k+1}^{r_{k}}\left(c_{k}, r_{k}\right.$ known constants) such that $\left(\mu_{k}\right) h_{k} \dot{\phi}_{k}=m_{k} z_{k}$, where $m_{k} z_{k}$ is a linear combination of $z_{k}$ and $z_{k x}$ at $j_{k}$. The linear problem consisting of $\left(E_{k}^{*}\right)$, an initial condition, and the nonhomogeneous end conditions $\left(\lambda_{k-1}\right)$ and $\left(\mu_{k}\right)$ (at $x=N$ the transformed (homogeneous) end condition supplies $\mu_{n}$ ) has a solution $z_{k}\left(\theta_{k}, \theta_{k+1} ; x, t\right)\left(z_{n}\right.$ involving only $\left.\theta_{n}\right)$. By substituting these $n$ functions $z_{k}$ into the transformed end condition at $x=0$ (which involves $\theta_{1}$ if the feed-concentration is variable), and into the $n-1$ continuity conditions at $j_{k}$, one obtains $n$ nonlinear functional equations for the $n$ functions $\theta_{k}$. Vice versa, solutions $\theta_{k}$ of these equations, if available, would yield via $z_{k}$ solutions $u_{k}$ of the original problem. (Received October 15, 1953.)

\section{W. R. Mann (p) and J. F. Blackburn: A steady state nonlinear heat problem.}

The problem is to find a function, $u(x, y)$, which satisfies Lapiace's equation in the semi-infinite strip, $y \geqq 0,0 \leqq x \leqq \pi$; which vanishes along the two vertical sides, and which satisfies the nonlinear condition $u_{y}(x, 0)=-G[u(x, 0)]$ along the horizontal side. $G$ is a continuous decreasing function which vanishes when $u=1$. The method of approach is to reduce the problem, by means of the finite Fourier transform, to the nonlinear integral equation $f(x)=(1 / \pi) \int_{0}^{\pi} K(x, \lambda) G[f(\lambda)] d \lambda$, where $K(x, \lambda)$ $=\log [1-\cos (x+\lambda) / 1-\cos (x-\lambda)]$ and where $f(x)=U(x, 0)$. The main results are proofs of the existence and uniqueness of $f(x)$ and the fact that $0 \leqq f(x) \leqq 1$. An iterative approximation to $f(x)$ is also discussed. (Received October 13,1953.)

\section{2t. T. L. Saaty and S. S. Gass: Parametric objective function.}

In problems such as the linear programming problem where there is a linear function to be optimized (called the objective function), it is desirable to study the behavior of solutions when the (cost) coefficients in the objective function are allowed to change. The problem is then to find the $x_{i}(j=1,2, \cdots, n)$ which minimize the linear form $\sum_{j=1}^{n}\left(c_{j}+\lambda c_{j}^{\prime}\right) \dot{x}_{j}$ and satisfy the constraints $x_{j} \geqq 0$ and $\sum_{j=1}^{n} a_{i j} x=a_{i 0}$ $(i=1,2, \cdots, m)$, where $c_{j}, c_{j}^{\prime}, a_{i j}$, and $a_{i 0}$ are constants (with at least one $c_{j} \neq 0$ ) and $\lambda$ a parameter. Using the Simplex method, a computational procedure is described which enables one, given a feasible solution, to determine the values of the parameter (if any) for which the solution minimizes the objective function; and given a minimum feasible solution how one can generate by the Simplex algorithm all minimum feasible solutions and the corresponding values of the parameter. To a minimum feasible solution there corresponds one connected set of $\lambda$ called a maximal determined range. Two maximal determined ranges intersect in at most one point and the set of maximal ranges is connected. The geometry of the problem is discussed in terms of the dual problem and an illustrative example is given. (Received October 2, 1953.) 


\section{GEOMETRY}

83t. M. K. Fort, Jr.: A cylindrical curve with maximum length and maximum height.

In a recent paper, Curves encircling a cylinder, Amer. Math. Monthly vol. 60 (1953) pp. 30-31, J. W. Green considers curves $C$ which have diameter 2 and which encircle a right circular cylinder of unit radius. Each such curve $C$ has a length $L(C)$ and a height $H(C)$. $(H(C)$ is defined to be the minimum distance between two planes perpendicular to the cylinder and containing $C$ between them.) Green proves that $2 \pi \leqq L(C) \leqq 2^{3 / 2} \pi$ and $0 \leqq H(C) \leqq 2^{1 / 2}$. In the present paper, a curve $C$ is constructed for which $L(C)=2^{3 / 2} \pi$ and $H(C)=2^{1 / 2}$. This example answers two questions raised by Green in his paper. (Received September 4, 1953.)

\section{C. M. Petty: Centroid surfaces.}

In $E^{(n)}$, let $M$ be given in polar coordinates $\rho, u$ by $0 \leqq \rho \leqq r(u), u \in \Omega_{n}$, where $\Omega_{n}$ is the surface of the $n$-dimensional unit sphere and $r(u)$ is a positive, even, continuous function defined on $\Omega_{n}$. The centroids of the half-volumes of $M$ bounded by hyperplanes through the origin $z$ (center of $M$ ) constitute a convex surface $C^{*}$ of class $C^{(2)}$ with center $z$. The surface $C^{*}$ is called the centroid surface of $M$ and determines $M$ uniquely. If $C^{*}$ is expanded by the factor $(n+1) K_{n} / 2 K_{n-1}\left(K_{r}=\pi^{r / 2} / \Gamma(r / 2+1)\right)$ we obtain the expanded centroid surface $C$ of $M$. One has the inequality $|C| \geqq|M|$ between the volumes where the equality holds only if $M$ is an ellipsoid. The volume $|M|$ of $M$ can be expressed by (1) $|M|^{n-1}=(1 / n)\left(K_{n}^{n-2} / K_{n-1}^{n}\right) \int \Omega_{n}|M(u)|{ }^{n-1}|C(u)| d \omega_{u}$, where $M(u)$ is the cross-section of $M$ with a hyperplane $H$ through $z$ perpendicular to $u$ and $C(u)$ is the expanded centroid surface of $M(u)$ in $H$. The relation (1) is a special case of a more general relation involving $(n-1)$ bodies of the type considered above. (Received October 21, 1953.)

\section{Topology}

\section{C. V. Aucoin: On skew rings.}

A skew ring consists of a set $R$ and two binary operations " * " and "०" such that $\{R, *\}$ forms a group and the operation "o" is two-sided distributive. If the operation " $*$ " is abelian then one has a ring without the associative law. This paper proves the following three theorems: 1 . If $\{R, *\}$ is a simple group, then either $R$ is isomorphic with the ordinary field of integers $\bmod$ a prime, or $R$ is trivial (i.e. $x \circ y=e$ for all $x$ and $y$ of $R$ ). 2. If $R$ is a finite skew ring and $I$ is the set of trivial elements (i.e. $I=\{x \mid x \circ r=r \circ x=e$ for each $r \in R\}$ ), then $R / I$ is isomorphic with the direct product $R_{1} \otimes R_{2} \otimes \cdots \otimes R_{n}$ of rings $R_{i}$ whose basic groups are abelian p-groups. 3. A compact connected topological skew ring satisfying the second countability axiom is trivial. (Received October 19, 1953.)

\section{C. E. Capel: Inverse limits of arcs and simple closed curves.}

Let $\left\{X_{\lambda}, \Pi_{\lambda \mu}, \Lambda\right\}$ be an inverse system of compact Hausdorff spaces in which the bounding maps, $\Pi_{\lambda \mu}$, are monotone; let $X=\lim _{\leftarrow}\left\{X_{\lambda}, \Pi_{\lambda \mu}, \Lambda\right\}$ be the inverse limit space. If each $X_{\lambda}$ is locally connected, then $X$ is locally connected. If each $X_{\lambda}$ is an arc (a simple closed curve) and $\Lambda$ is countable, then $X$ is an arc (a simple closed curve). (Received October 13, 1953.) 


\section{H. S. Collins: $A$ new class of complete bornologique spaces.}

Recent extensions of the closed graph theorem have aroused considerable interest in a certain class of locally convex linear togological spaces; namely, the complete bornologique spaces. It is the purpose of this note to show that certain additional function spaces belong to this class. Let $S$ be any locally compact Hausdorff topological space, and denote by $E$ the space of real (or complex) valued continuous functions on $S$, supplied with the compact-open topology. Let $E^{*}$ denote the space of continuous linear functionals on $E$, supplied with the strong topology. Our result is that $E^{*}$ is a complete bornologique space when $S$ is paracompact. Previous results along these lines required that $S$ be sigma-compact. (Received October 13,1953.)

88. D. O. Ellis: On the topolattice and permutation group of an infinite set. II.

Let $S$ be an infinite set and $\Lambda$ be the lattice of all $T_{1}$ topologies on $S . \Lambda$ is called the topolattice (Robert Bagley and David Ellis, On the topolattice and permutation group of an infinite set, Bull. Amer. Math. Soc. Abstract 60-1-89) of $S$. One shows: The group of permutations of $S$ is isomorphic to the group of automorphisms of $\boldsymbol{\Lambda}$. Any automorphism of $\Lambda$ is completely determined by its behavior on the hyperplanes of $\Lambda$. The methods used are lattice-theoretic. (Received September 3, 1953.)

89. D. O. Ellis and Robert Bagley (p): On the topolattice and permutation group of an infinite set.

Let $S$ be an infinite set and let $\Lambda$ denote the set of all $T_{1}$ topologies on $S$. We consider a permutation $f$ of $S$ and define $f(\alpha, \beta)=0$ for $\alpha, \beta \in \Lambda$ when $f$ is continuous with $\alpha$ as domain topology and $\beta$ as range topology in $S$, and $f(\alpha, \beta)=1$ otherwise. We then find a necessary and sufficient set of algebraic conditions on a general mapping of $\Lambda \otimes \tilde{\Lambda}$ onto the two-element chain $(0,1)$ in order that the mapping be induced in the above way by a permutation of $S$. The methods are essentially lattice-theoretic. (Received June 16, 1953.)

90. D. O. Etter and J. S. Griffin, Jr. (p): On the metrizability of the bundle space.

It has been shown by Yu. M. Smirnov (see American Mathematical Society Translation No. 91, On the metrization of topological spaces) that if $X$ is a topological space, then $X$ is metrizable if and only if $X$ is paracompact and has an open cover each of whose members is metrizable. This leads immediately to the proposition: if $\{X, B, \pi, Y, \mathcal{V}, \phi, G\}$ is a fibre bundle whose base space $B$ and fibre $Y$ are metrizable, then the bundle space $X$ is also metrizable: since if $U \in \mathcal{U}$ then $\pi^{-1}(U)$ is metrizable, it suffices to show $X$ paracompact. But if $\mathcal{C}$ is any open cover for $X$, let (1) $\nu$ be a locally finite refinement of $\mathcal{V},(2) \mathcal{W}$ be a closure-refinement of $\boldsymbol{V}$ which is also locally finite, (3) $c$ be a function on $\mathscr{W}$ such that if $W \in \mathscr{W}$ then $c(W) \in \mathcal{V}$ and, $W \subset c(W)$, (4) $\mathcal{D}_{V}$ be the family of intersections of members of $\mathcal{C}$ with $\pi^{-1}(V)$, (5) $\mathcal{D}_{V}^{*}$ be a locally finite refinement of $D_{V}$ where $V \in \mathcal{V}$, (6) $\varepsilon_{W}$ be the family of intersections of members of $\mathcal{D}_{c}^{*}(W)$ with $\pi^{-1}(W)$ where $W \in \mathcal{W}$. Then $\bigcup_{W \in \mathcal{W}} \mathcal{E}_{W}$ is a locally finite open cover refining $C$. (Received October 15, 1953.)

91. W. M. Faucett: $A$ result on mobs irreducibly connected between two points. Preliminary report. 
Let $S$ be a compact connected Hausdorff space together with a continuous, associative multiplication. Further, let $S$ have a unit and be irreducibly connected between $a$ and $b$, where $a^{2}$ and $b^{2}$ are idempotents. Then $S$ is commutative if and only if $S$ contains an element $O$ such that $O x=O=x O$ for all $x \in S$. $S$ can be linearly ordered and in the course of the proof it is shown that the multiplication in $S$ is continuous with respect to the order topology. Also if $a$ and $b$ are the unit and the zero of $S$ respectively, and $S$ has no other idempotents, then $S$ contains a countable dense set. (Received October 13, 1953.)

\section{M. K. Fort, Jr.: A theorem about topological n-cells.}

The main result obtained in this paper is: If $G$ is a nonempty, open, connected subset of Euclidean $n$-space, then there exists a sequence $E_{1} E_{2} E_{3} \cdots$ of closed topological $n$-cells such that ${ }_{j 1} E_{j} G$. As a corollary to this result we obtain: If $G$ is a nonempty, open, connected subset of Euclidean $n$-space, then there exists a subset $H$ of $G$ such that: dimension of $H$ is less than $n, G-H$ is open and connected, all of the homotopy groups of $G-H$ vanish. (Received August 10, 1953.)

\section{H. C. Griffith: The enclosing of cells in 3-space by simple closed} surfaces.

Harrold's property $P$ for arcs [Bull. Amer. Math. Soc. Abstract 59-4-434] is extended to apply to closed $k$-cells in 3-space for $k=1,2$, or 3 . If $E^{1}$ is a segment and $E^{k}=E^{1} \times E^{k-1}$, then a $k$-cell $C$ is the image of $E^{k}$ under a homeomorphism $h$ and $\mathcal{T}_{h}$ denotes the collection of sets $h\left(x \times E^{k-1}\right)$. $C$ has the uniform disk property relative to $\mathcal{T}_{h}$ provided there is a positive $\delta$ such that for each positive $\epsilon$ and $T \in \mathcal{T}_{h}$ there is a 2-cell $D$ such that (1) $d(C$, Boundary of $D)>\delta,(2) D \cap C$ is a $(k-1)$-cell, (3) $D$ is locally polyhedral except on $C,(4) \rho(T, D \cap C)<\epsilon$ (where $\rho$ is an appropriate metric on $\mathcal{G}_{h}$ ), and (5) $D$ meets $C$ essentially. Theorem. A cell $C$ with property $\mathrm{P}$ is enclosed by a polyhedral 2-sphere $K_{\epsilon} C S(C, \epsilon)$ for every positive $\epsilon$ if and only if it has the uniform disk property relative to $\mathcal{T}_{h}$. (Received October 14, 1953).

\section{R. J. Koch: Remarks on primitive idempotents in compact mobs} with zero.

Recall that a mob is a Hausdorff space together with a continuous associative multiplication. Denote by $X$ a compact mob with zero, let $N$ be the subset of elements whose powers converge to 0 , and denote by $E_{p}$ the set of primitive idempotents of $X$. It is shown that if $e$ is a nonzero primitive idempotent of $X$ then $X e X$ is a minimal non-nil ideal. This is the converse of a result of Numakura (Bulletin of the Yamagata University, no. $4 ; 1951$ ), and is proved by showing these are equivalent: (1) $e$ is a nonzero primitive idempotent. (2) $X e$ is a minimal non-nil left ideal. (3) $(e X e) \backslash N$ is a (compact) group. (4) $e X e$ is a minimal non-nil bi-ideal (Bull. Amer. Math. Soc. Abstract 58-6-575). From Numakura's results it then follows that $\left(X E_{p} X\right) \backslash N=\left(X E_{p}\right) \backslash N$ is the disjoint union of (compact) groups. Finally, it is shown that if $X$ is connected, then $\left(X E_{p}\right) \cap N$ is dense in $X E_{p}$. (Received October 13,1953.)

95t. R. J. Koch: Theorems on mobs.

Recall that a mob is a Hausdorff space together with a continuous associative multiplication. If $e$ is an idempotent of the semigroup $X$ then $X e$ is a minimal left ideal iff $e X e$ is a group. If $X$ is a semigroup containing minimal left ideals and minimal right ideals, then the Suschkewitsch kernel $K$ is a group iff $K$ meets a normal set. 
The center of $K$ is the intersection of $K$ with the center of $X$. A natural partition of $X$ is exhibited which separates the maximal subgroups of $K$, and if $X$ is a compact mob this decomposition is upper semicontinuous. It is shown that if a left translate of some closed subset of the compact mob $X$ exhausts $X$, then $X$ has a left unit. A mob is termed monothetic if it has a dense cyclic submob. Among results established for compact monothetic mobs is the fact that a compact monothetic mob with two distinct generators is a group. A locally compact monothetic mob with kernel is compact. A locally compact monothetic zero-dimensional mob with unit is a compact group. Two generalizations of monothetic mobs are considered. Finally, a quotient structure is discussed for compact mobs which contain a closed normal set. (Received October 13, (1953.)

96. L. F. McAuley: Generalized upper semi-continuous collections as applied to aposyndetic decompositions of continua.

Suppose that $G$ is a collection of point sets. If $\left\{x_{i}\right\}$ is a sequence of points converging to a point $x, x \in g \in G, x_{i} \in g_{i} \in G$ for each $i, g_{i} \neq g_{j}$ for $i \neq j$, and if $\left\{y_{i}\right\}$ is a sequence of points, $y_{i} \in g_{i}$ for each $i$; then every convergent subsequence of $\left\{y_{i}\right\}$ converges to a point of $g$. $G$ is said to be an upper semi-continuous collection. Certain subcollections of $G$ are defined to be "regions." Suppose that $M$ is a continuum. Let $H$ denote the collection of all point sets $M(p)$ for $p \in M$ (see Bull. Amer. Math. Soc. Abstract 59-1-77). $S(p, x)$ denotes the set of all points $y$ of $M(p)$ such that there does not exist a subcollection $N$ of $H$ such that $N^{*}$ is the sum of a finite number of continua and $N^{*}$ separates $x$ from $y$ in $M$. Suppose that $M$ is also a completely separable semi-metric topological space and that $K$ is the collection of all point sets $S(p, x)$ for each point $p$ of $M$ and each point $x$ of $M(p)$; then $K$ is an upper semi-continuous collection of mutually exclusive closed point sets filling up $M$; and furthermore, with respect to its elements as points, $K$ is an aposyndetic, connected, and completely separable Hausdorff topological space. (Received October 14, 1953.)

\section{Frances A. Norton: Mayer-Vietoris sequence for homotopy} groups.

Let $X$ be a fibre space over $B$ with fibre $F$. Suppose that $B$ is a topological product $B_{1} \times B_{2}$. For $j=1,2$, the inclusion map $i_{j}: B_{j} \rightarrow B$ gives an induced fibre space $X_{j}$ over $B_{i}$ with the same fibre $F$. Assuming $X, B, F$ to be arc-wise connected, it is shown that there exists an exact sequence of the form $\cdots \rightarrow \pi_{n}(F) \rightarrow I_{n}\left(X_{n}\right)+\pi_{n}\left(X_{2}\right)$ $\rightarrow{ }^{J} \pi_{n}(X) \rightarrow{ }^{\Delta} \pi_{n-1}(F) \rightarrow \cdots$ where $I, J, \Delta$ are defined, in a natural manner, by means of some injection homomorphisms and boundary homomorphisms. This sequence of homotopy groups corresponds to the well known Mayer-Vietoris sequence in homology theory. (Received October 19, 1953.)

\section{R. A. Roberts: Duality theorems for generalized manifolds.}

Two topologies are defined for the Cech homology groups using field coefficients, one for locally compact spaces and the other for compact spaces. These topologies enable the author to prove Alexander and Poincaré type duality theorems for generalized manifolds, without restrictions such as perfect normality, and to obtain some preliminary results concerning carriers of Cech cycles. For example, it is shown that if $M$ is a compact space and $F$ is an algebraic field, then there is a topology for $H^{\circ}(M, F)$ and a collection of pairs of points of $M$ such that the o-homology classes 
of the $o$-Cech cycles carried by each pair of points have $H^{\circ}(M, F)$ as their linear closure in the topology. (Received October 13, 1953.)

99. W. L. Strother: Fixed points in the space of subsets.

It is shown that if $X$ is a compact Hausdorf space then $S(X)$ has the fixed point property for functions which are embedded extensions of functions on $X$ to $S(X)$. The proof is based on continuity for certain associated multivalued functions. (Received October 13, 1953.)

\section{P. M. Swingle: Locally $n$-divisible and related connexes.}

New types of connexes related to those in Generalization of biconnected sets (Amer. J. Math. vol. 53 (1931) pp. 385-400) are given. For example a connexe $M$ is locally $n$-divisible at a point $x$ if and only if, for each region $H, x \in H$, (1) there exists a subregion $R$ of $H, x \in R$, and $n$ subconnexes $M_{i}$ of $M$, mutually exclusive and each dense in $R$, and $M \cdot R=\left(M_{1}+\cdots+M_{n}\right) \cdot R$; and (2) if the $n$ subconnexes $M_{i}^{\prime}$ of $M$ are such that the $M_{i}^{\prime} \cdot R$ are mutually exclusive and each dense in $M \cdot R$, then there does not exist a subconnexe $M_{n+1}^{\prime}$ of $M$, mutually exclusive with the $M_{i}^{\prime}$ 's in $M \cdot R$, such that $R \cdot M_{n+1}^{\prime} \neq 0$ and $M \cdot R=\left(M_{1}^{\prime}+\cdots+M_{n+1}^{\prime}\right) \cdot R$. Various existence theorems for these connexes are proven including: There exists in any region $T$ of the plane a widely connected subset $M$, which, for $n$ an integer $>0$, is locally $n$-divisible and $M$ and $T$ have the same closure; For $n>1$ there exists in the plane an $n$-biconnexe confined connexe, which contains a subconnexe $M_{e}$ which does not contain a biconnected subset. (Received October 13, 1953.)

\section{A. D. Wallace: Peripheral subgroups of clans.}

Recall that a clan is a compact connected Hausdorff space together with a continuous associative multiplication with unit. If $u$ is the unit of the clan $S$ and if $H(u)$ is the set of elements of $S$ with inverses, then $H(u)$ is a compact (topological) subgroup of $S$. It is known that, if $S$ is contained in $R^{n+1}, n \geqq 1$, then $H(u) \subset F(S)$, the set theoretic boundary of $S$. Simple examples show that $H(u)$ need not coincide with $F(S)$. In this paper this theorem is proved: Let $S$ be a clan contained in $R^{n+1}, n \geqq 1$, and let $S$ not cut $R^{n+1}$. If $H(u)$ cuts $R^{n+1}$, then $H(u)=F(S)$ and $H(u)$ irreducibly cuts $R^{n+1}$ into the two components $R^{n+1} / S$ and $S / H(u)$. Moreover $H(u)$ is a compact connected Lie group. The condition " $H(u)$ cuts $R^{n+1}$ " can be replaced by "dim $H(u)$ $=n: "$ (Received September 1, 1953.)

W. M. WhYBURN, Associate Secretary 